\title{
When the chest is clueless, look downstairs
}

\author{
D. Attinà1 ${ }^{1}$, F. Ciccarese ${ }^{1}$, M. Mughetti ${ }^{1}$, A. Rocca 2 , P. Contini2, M. Zompatori ${ }^{1}$
}

A 39-year-old male, never smoker, with no past or family history of pulmonary diseases, was admitted to the emergency department complaining of cough and progressively worsening dyspnoea.

Chest X-ray showed a right-sided pneumothorax (figure 1) and a pleural tube was placed. One month later high resolution computed tomography (HRCT) was performed and showed a residual pneumothorax and multiple thin-walled cysts, randomly distributed in the lung parenchyma, especially in the lower lobes (figure $2 \mathrm{~A}-\mathrm{B}-\mathrm{C}$ ). In the lower scans, partly including the superior abdomen, a 2-cm-solid-lesion was found in the left kidney. A subsequent contrast CT scan of abdomen showed an evident contrast-enhancement (figure 2 D). The CT-guided biopsy confirmed the neoplastic nature of the lesion that was characterixed as an oncocytoma.

A genetic test revealed a mutation of Folliculin gene (FLCN): c.1285delC mutation in the exon 11. The same test was performed also on close relatives (parents) and father (80 years old, asymptomatic) resulted positive.

Birt-Hogg-Dubè (BHD) syndrome is a rare autosomal dominant disease, caused by germline mutations in FLCN gene, which is generally characterised by a typical triad, including skin fibrofolliculomas, lung cysts/ spontaneous pneumothorax and kidney tumors [1]. FLCN gene, discovered only in 2002, encodes a protein named Folliculin, which is expressed in skin tissue, nephrons and type I pulmonary alveolar epithelial cells [2]. Folliculin's functions have not been completely characterised, however they are thought to be involved in cell growth and tumour suppression (at least in kidney tumours, a somatic "second hit" mutation has been observed).

BHD syndrome has an incomplete penetrance and a wide phenotypic variability. In 1977 this syndrome was firstly described as a familiar dermatologic disorder, and it is characterised by fibrofolliculomas (multiple, whiteish, smooth, dome-shaped papules on the head, neck, face and upper trunk), associated with trichodiscomas (tumours of hair disk) and acro- chordons (skin tags) [3]. It was later demonstrated that BHD is not always associated with cutaneous manifestations (not observed in $25 \%$ of FLCN-mutation carriers) and can involve only lungs and kidneys. Typically, basal, subpleural or peri-vascular cysts and eventually spontaneous pneumothorax are observed [4], whereas in the kidney the most threatening manifestation is represented by a mixed oncoytic/cromophobe carcinoma, which is often multifocal and bilateral. However, other subtypes can occur, including clear-cell, papillary or tubular-papillary carcinoma [5]. In addition, other several clinical manifestations have been occasionally reported.

Typically, $80 \%$ of patients with BHD show multiple lung cysts and they present a 50-time increased risk to develop pneumothorax. Lung cysts are variable in size and shape. Lung function tests are usually normal. No relationship with smoking history has been described.

In conclusion, BHD is a genetic syndrome probably underestimated for several reasons: the gene was discovered only in 2002, skin lesions were considered the key finding for diagnosis but they are not always present, pulmonary manifestations are poorly understood

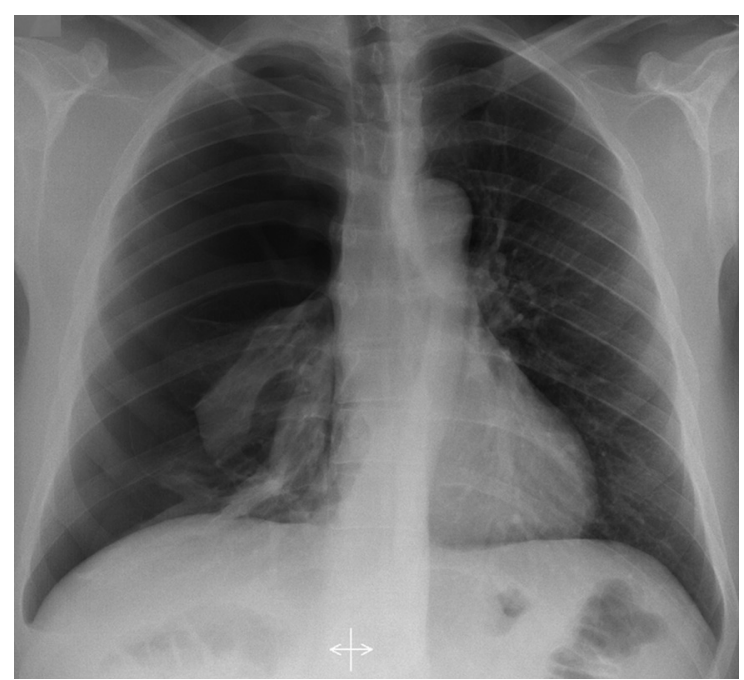

Fig. 1. - Chest Xray. Right-sided pneumothorax with almost complete right lung collapse and minimal amount of right pleural effusion in the lateral costophrenic recess.
Monaldi Arch Chest Dis 2013; 79: 1, 49-50

Keywords: Birth-Hogg-Dubè syndrome,

Pneumothorax,

Lung cysts, Kidney neoplasm, CT thorax.

1 Radiology Unit, Cardio-ThoracicVascular Department, University Hospital S. Orsola-Malpighi, Via Massarenti 9, 40128 Bologna: 2 Pneumology and Critical Care Respiratory Unit, Cardio-ThoracicVascular Department, University Hospital

S. Orsola-Malpighi, Via Massarenti 9 , 40128 Bologna, Italy.

Corrispondence: Domenico Attinà, Radiology Unit, Cardio-ThoracicVascular Department, University Hospital S. Orsola-Malpighi, Via Massarenti 9. 40128 Bologna, Italy;e-mail: dome.attina@gmail.com 

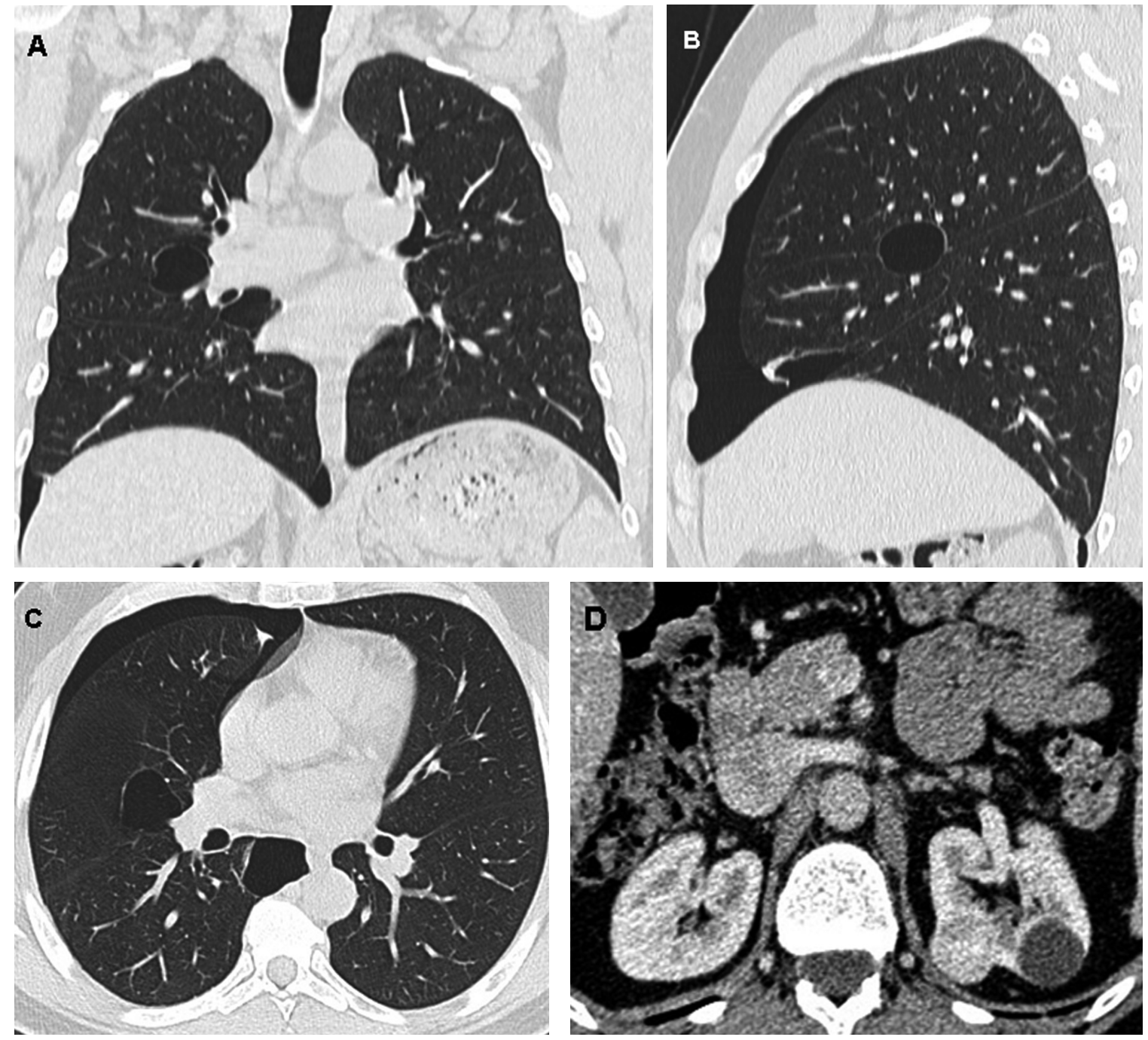

Fig. 2. - CT figures A-B-C show the persistence of a moderate right-sided pneumothorax and multiple thin-walled, subpleural, air-filled lung cysts. Figure D shows the presence of one cortical renal cyst and a solid lesion with an elevated contrastenhancement in the left kidney. This lesion proved to be an oncocytoma after the CT-guided biopsy.

and the disease is not necessarily symptomatic. Spontaneous pneumothorax, can also be the first and the only clinical manifestation. Last but not least, generally the HRCT scans are tailored to cover only the lung volume, to reduce radiation exposure but, when serendipitously the upper part of the abdomen is included in the CT volumetric acquisition, these images should always be carefully examined with abdominal or mediastinal window settings, because, when the chest is clueless, taking a good look downstairs can be very rewarding.

\section{References}

1. Menko FH, van Steensel MA, Giraud S, FriisHansen L, et al. European BHD Consortium. Birt-
Hogg-Dubé syndrome: diagnosis and management. Lancet Oncol 2009; 10: 1199-1206.

2. Nickerson ML, Warren MB, Toro JR, Matrosova $\mathrm{V}$, et al. Mutations in a novel gene lead to kidney tumors, lung wall defects, and benign tumors of the hair follicle in patients with the Birt-Hogg-Dubé syndrome. Cancer Cell 2002; 2: 157-164.

3. Birt AR, Hogg GR, Dubé WJ. Hereditary multiple fibrofolliculomas with trichodiscomas and acrochordons. Arch Dermatol 1977; 113: 1674-1677.

4. Toro JR, Pautler SE, Stewart L, Glenn GM, et al. Lung cysts, spontaneous pneumothorax, and genetic associations in 89 families with Birt-Hogg-Dubé syndrome. Am J Respir Crit Care Med 2007; 175: 1044-1053.

5. Pavlovich CP, Grubb RL, Hurley K, Glenn GM, et $a l$. Evaluation and management of renal tumours in the Birt-Hogg-Dubé syndrome. J Urol 2005; 173: 1482-1486. 